\title{
Entanglement Between Nanoelectromechanical Systems Mediated by a Transmission Line Resonator
}

Olimpio Pereira de Sa Neto ( $\square$ olimpioqedc@gmail.com )

Universidade Estadual do Piaui https://orcid.org/0000-0003-0215-249X

\section{Research Article}

Keywords: Nanoelectromechanical, Systems, Transmission Line Resonator, Bipartite Entanglement

Posted Date: February 1st, 2022

DOI: https://doi.org/10.21203/rs.3.rs-1293209/v1

License: (c) (i) This work is licensed under a Creative Commons Attribution 4.0 International License.

Read Full License 


\title{
Entanglement Between Nanoelectromechanical Systems Mediated by a Transmission Line Resonator
}

\author{
O. P. de Sá Neto ${ }^{a, b}$
}

Received: date / Accepted: date

\begin{abstract}
In this letters, is considered a simple chip composed of three devices, where we have two nanoelectromechanical systems capacitive coupling with a transmission line resonator, in which this interaction is approximated to two non-degenerate parametric oscilatior interaction. For Entanglement analysis, we take two identical nanoelectromechanical systems with a thermal initial state.
\end{abstract}

Keywords Nanoelectromechanical · Systems, Transmission Line Resonator · Bipartite Entanglement

\section{Introduction}

Quantum processing information require normally the manipulation of entangled states as well as the investigation of the entanglement shared between distant sites [1-4]. In fact, it has been exploited extensively - both experimental and theoretical studies - using trapped atomic ions [5], entangled photons [28] and cavity QED [7] providing suitable scenarios for this propose. On the one hand, such quantum systems inevitably interact with the surrounding environment generating effects of decoherence. Several proposals have been developed for minimizing, or using the environment to control the entangled states theses small quantum system. On the other hand, the manipulation of quantum entangled states can be realized more efficiently using

O. P de Sá Neto

${ }^{a}$ Coordenação de Ciências da Computação, Universidade Estadual do Piauí, cep: 64.202-220, Parnaíba (PI), Brazil

${ }^{b}$ Instituto de Física, Universidade Federal do Rio de Janeiro,

Universidade Federal do Rio de Janeiro, (RJ), 21942-972,

Brazil Tel.: +55-86-981677387

Fax: +55-86- 33211800

E-mail: olimpioqedc@gmail.com
Gaussian operations (operations that keep the Gaussian character) [8]. Gaussian states (GS) have attracted much attention due to facility in prepare and manipulate resources for processing of quantum information [9]-[17]. The investigation of the entanglement features using symplectic and Lorentz groups in a two-mode bipartite GS was explored in Ref.[10]. The tight bounds for the entanglement in two-mode GS employing the properties of Gaussian channels was studied in Ref.[8]. For the case of an arbitrary two-mode GS dissipating in local Gaussian environments has been discussed in other works [11]. Therefore it would be interesting to extend our research for study the dynamics of entanglement between two ions (considering only the vibrational degrees of freedom) using two-mode bipartite GS.

Here we propose a new architecture to model two nondegenerate parametric amplifier through the chip that consists of a Transmission Line Ressonator (TLR) capacitively coupled to two nanoelectromechanical systems (NEMS), we evaluate the entanglement via symplectic [8], and the enhanced of the non-separability of these states in time function through of the radiation intensity of the TLR. This paper is organized as follows: In Sec. 2 we define the notation used for the Gaussian bipartite states and review a form for evaluate the symplectic eigenvalues of the covariance matrix [8]. Sec. 3 contains the theoretical model of the our investigation and we show, a case, how to generate a nondegenerate parametric oscillator type. The main results are show in Sec. 4 and 5, among them we can highlight the improvement of the entanglement time and your measure. In Sec. 6 we summarize all the find out obtained in this work. 


\section{Gaussian Bipartite States}

In the quantum mechanics, gaussian states are ones that are completely characterized by second order moments. Thus, the information on GS can be stored in mean values vectors and covariance matrices [10]. On bipartite systems described by bosonic annihilation $\left(\hat{b}_{1}\right.$, $\left.\hat{b}_{2}\right)$ and creation $\left(\hat{b}_{1}^{\dagger}, \hat{b}_{2}^{\dagger}\right)$ operators, the Covariance Matrix $(V)$ can be represented by [10],

$V=\left(\begin{array}{cc}V_{1} & C \\ C^{\dagger} & V_{2}\end{array}\right)$

with

$V_{i}=\left(n_{i}+\frac{1}{2}\right) \mathcal{I}, \quad C=\left(\begin{array}{c}m_{s} m_{c} \\ m_{c}^{*} m_{s}^{*}\end{array}\right)$,

where

$$
\begin{aligned}
& n_{1}=\operatorname{Tr}\left\{\hat{b}_{1}^{\dagger} \hat{b}_{1} \rho\right\}, \quad m_{c}=-\operatorname{Tr}\left\{\hat{b}_{1} \hat{b}_{2} \rho\right\}, \\
& n_{2}=\operatorname{Tr}\left\{\hat{b}_{2}^{\dagger} \hat{b}_{2} \rho\right\}, \quad m_{1}=-\operatorname{Tr}\left\{\hat{b}_{1}^{2} \rho\right\}, \\
& m_{s}=\operatorname{Tr}\left\{\hat{b}_{1} \hat{b}_{2}^{\dagger} \rho\right\}, \quad m_{2}=-\operatorname{Tr}\left\{\hat{b}_{2}^{2} \rho\right\}
\end{aligned}
$$

for $n_{1}, m_{s}, m_{c} \in \mathcal{R}, V_{1}$ and $V_{2}$ being hermitian matrices containing only local values [10]. We should notice that general covariance matrices for two mode systems can be brought to this form by local unitary transformations. Gaussian operations preserve an input GS character, in other words, Gaussian operations are an entirely positive map acting on the corresponding density operators [14]. The covariance matrix assumes important values for the bipartite systems and these values provide local and global properties. The local symplectic invariants can be defined by:

$$
I_{1}=\operatorname{det} V_{1}, \quad I_{2}=\operatorname{det} V_{2}
$$$$
I_{3}=\operatorname{det} C, I_{4}=2\left|I_{3}\right| \sqrt{I_{1} I_{2}},
$$

stands for the group $S p(2, R) \otimes S p(2, R)[8, ?]$. Finally, with local symplectic invariants demonstrated in (3) we can calculate the symplectic eigenvalues [8] of the covariance matrix (1)

$\eta_{ \pm}^{2}=\frac{I_{1}+I_{2}}{2}+I_{3} \pm \sqrt{\left(\frac{I_{1}-I_{2}}{2}\right)^{2}+\left(I_{1}+I_{2}\right) I_{3}+I_{4}}$

Therefore, serving as criterion to define physical states $\left(\eta_{+}>\eta_{-}\right)$, entanglement states $\left(\eta_{-}<0,5\right)$ and separable states $\left(\eta_{-} \geq 0,5\right)$. This expression will serve to obtain the results of this work according to our model formulated in the following section.

\section{Physical System}

Considering the system in chip how figure (1), w hava a TLR capacitive coupled with two NEMS can be characterized by the following Hamiltonian

$H=H_{0}+H_{e}$,

$$
H_{0}=\frac{\Phi^{2}}{2 L}+\frac{Q^{2}}{2 C}+\sum_{j=1,2}\left(\frac{p_{j}^{2}}{2 m_{j}}+\frac{1}{2} m_{j} \nu_{j}^{2} x_{j}^{2}\right),
$$

where the $L, C, \Phi$, and $Q$ are the characteristic inductance, the characteristic capacitance, canonical coordinates for TLR representing magnetic flux and charge in a single mode, analogous to an LC circuit. The $m_{j}, \omega_{j}$, $p_{j}$, and $x_{j}$ are the masses, the frequencies of oscillations, canonical the moments and positions of each NEMS, respectively. The electrostatic interaction energy $\left(H_{e}\right)$ is given by [27]

$$
H_{e}=\sum_{j=, 1,2} \frac{Q^{2}}{2 C\left(x_{j}\right)}=\frac{1}{2 \epsilon_{0} A} Q^{2} \sum_{j=1,2}\left(x_{j}+d\right)
$$

where $C\left(x_{j}\right)=\frac{\epsilon_{0} A}{d+x_{j}}$ is the capacitance between TLRNEMS in function of the position of the NEMS, with $d$ is equilibrium and separation TLR-NEMS. Now rewriting the Hamiltonian (5) as follows,

$$
\begin{aligned}
H & =\frac{\Phi^{2}}{2 L}+\left(\frac{1}{2 C}+\frac{d}{2 \epsilon_{0} A}\right) Q^{2} \\
& +\sum_{j=1,2}\left(\frac{p_{j}^{2}}{2 m_{j}}+\frac{1}{2} m_{j} \nu_{j}^{2} x_{j}^{2}\right)+\frac{1}{2 \epsilon_{0} A} Q^{2} \sum_{j=1,2} x_{j} .
\end{aligned}
$$

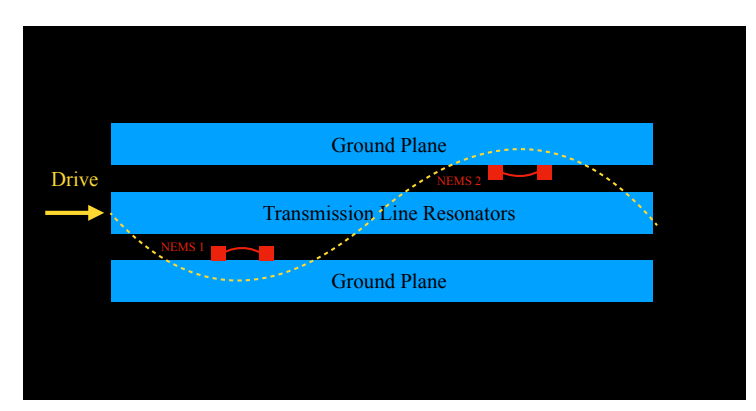

Fig. 1 Schematic Circuit.

To quantize the system, we define the operators $\left(Q(t) \rightarrow \hat{Q}(t), \Phi(t) \rightarrow \hat{\Phi}(t), x_{j}(t) \rightarrow \hat{x}_{j}(t)\right.$ and $p_{j}(t) \rightarrow$ $\left.\hat{p}_{j}(t)\right)$ of charge, magnetic flux positions and moments of TLR, NEMS 1 and 2 in terms of operator creation $\left(\hat{a}^{\dagger}, \hat{b}_{1}^{\dagger}, \hat{b}_{2}^{\dagger}\right)$ and annihilation $\left(\hat{a}, \hat{b}_{1}, \hat{b}_{2}\right)$,

$$
\begin{gathered}
\hat{Q}=\sqrt{\frac{\hbar \omega \tilde{C}}{2}}\left(\hat{a}+\hat{a}^{\dagger}\right), \hat{\Phi}=i \sqrt{\frac{\hbar \omega L}{2}}\left(\hat{a}^{\dagger}-\hat{a}\right), \\
\hat{x}_{j}=\sqrt{\frac{\hbar}{2 m_{j} \nu_{j}}}\left(\hat{b}_{j}+\hat{b}_{j}^{\dagger}\right), \hat{p}_{j}=i \sqrt{\frac{\hbar m_{j} \nu_{j}}{2}}\left(\hat{b}_{j}^{\dagger}-\hat{b}_{j}\right),
\end{gathered}
$$

for $i=1,2, \hbar$ is the Plank constant, $\frac{1}{\tilde{C}}=\frac{1}{C}+\frac{d}{\epsilon_{0} A}$, and $\omega^{2}=L \tilde{C}$.

Thus, the Hamiltonian to system is given by

$\hat{H}=\hat{H}_{0}+\hat{H}_{e}$, 
with

$\hat{H}_{0}=\hbar \omega \hat{a}^{\dagger} \hat{a}+\hbar \sum_{j=1,2} \nu_{j} \hat{b}_{j}^{\dagger} \hat{b}_{j}$

$\hat{H}_{e}=\hbar \sum_{j=1,2} \sqrt{\frac{\hbar \omega^{2} \tilde{C}^{2}}{8 m_{j} \nu_{j}}}\left(\hat{a}+\hat{a}^{\dagger}\right)^{2}\left(\hat{b}_{j}+\hat{b}_{j}^{\dagger}\right)$

Applying the first rotation $U_{1}(t)=\exp \left[-i \omega \hat{a}^{\dagger} \hat{a} t\right]$ in the Hamiltonian (7), we have (with $m=m_{1}=m_{2}$ $\left.\nu=\nu_{1}=\nu_{2}, g=\sqrt{\frac{\hbar \omega^{2} \tilde{C}^{2}}{8 m \nu}}\right)$

$\hat{H}_{1}=\hbar \nu \sum_{j=1,2} \hat{b}_{j}^{\dagger} \hat{b}_{j}+\hbar g \sum_{j=1,2}\left(\hat{a} e^{-i \omega t}+\hat{a}^{\dagger} e^{i \omega t}\right)^{2}\left(\hat{b}_{j}+\hat{b}_{j}^{\dagger}\right)$,

we are in the rotating wave approximation (RWA)

$\hat{H}_{1}=\hbar \omega \hat{a}^{\dagger} \hat{a}+\hbar \nu \sum_{j=1,2} \hat{b}_{j}^{\dagger} \hat{b}_{j}+\hbar g \sum_{j=1,2} \hat{a}^{\dagger} \hat{a}\left(\hat{b}_{j}+\hat{b}_{j}^{\dagger}\right)$.

Considering a drive intense in the TLR, we can linearize the interaction [18], give $\hat{a} \approx \sqrt{\bar{n}}+\hat{\alpha} e^{-i \Omega t}$, $\langle\hat{a}\rangle=\sqrt{\bar{n}}$, where $\Omega$ is drive frequency, we have

$$
\begin{aligned}
\hat{H}_{1} & =\hbar \omega\left(\sqrt{\bar{n}}+\hat{\alpha} e^{-i \Omega t}\right)^{\dagger}\left(\sqrt{\bar{n}}+\hat{\alpha} e^{-i \Omega t}\right)+\hbar \nu \sum_{j=1,2} \hat{b}_{j}^{\dagger} \hat{b}_{j} \\
& +\hbar g\left(\sqrt{\bar{n}}+\hat{\alpha} e^{-i \Omega t}\right)^{\dagger}\left(\sqrt{\bar{n}}+\hat{\alpha} e^{-i \Omega t}\right) \sum_{j=1,2}\left(\hat{b}_{j}+\hat{b}_{j}^{\dagger}\right) .
\end{aligned}
$$

Applying the second rotation

$U_{2}(t)=\exp \left[-i\left(\delta \hat{\alpha}^{\dagger} \hat{\alpha}+\nu \sum_{j=1,2} \hat{b}_{j}^{\dagger} \hat{b}_{j}\right) t\right]$

in (9), where $\delta$ is the detuning between the drive frequency and TLR $\delta=\Omega-\omega$ (illustrated in figure 1), finally making RWA with $\delta \approx-\nu$, we get the effective Hamiltonian

$\hat{H}_{e f f}=\hbar g \sqrt{\bar{n}} \sum_{j=1,2}\left(\hat{\alpha} \hat{b}_{j}+\hat{\alpha}^{\dagger} \hat{b}_{j}^{\dagger}\right)$

characterizing two non-degenerate parametric oscillate, an acceptable regime in intarations via radiation pressure [18] and electromechanical circuit [19]. For quantum total domain situations $(\hbar \omega \gg k T)$, the equations of motion are

$$
\begin{aligned}
\partial_{t} \hat{\alpha} & =-i g \sqrt{\bar{n}} \hat{b}_{1}^{\dagger}-i g \sqrt{\bar{n}} \hat{b}_{2}^{\dagger}, \\
\partial_{t} \hat{b}_{1}^{\dagger} & =i g \sqrt{\bar{n}} \hat{\alpha}, \\
\partial_{t} \hat{b}_{2}^{\dagger} & =i g \sqrt{\bar{n}} \hat{\alpha} .
\end{aligned}
$$

Therefore, the solutions are

$$
\begin{aligned}
\hat{\alpha}(t) & =\hat{\alpha}(0) \mathcal{W}(t) \\
& -i \frac{\sqrt{2}}{2}\left(\hat{b}_{1}^{\dagger}(0)+\hat{b}_{2}^{\dagger}(0)\right) \mathcal{Z}(t), \\
\hat{b}_{1}^{\dagger}(t) & =i \frac{\sqrt{2}}{2} \hat{\alpha}(0) \mathcal{Z}(t)+\hat{b}_{1}^{\dagger}(0)\left[\frac{\mathcal{W}(t)+1}{2}\right] \\
& +\hat{b}_{2}^{\dagger}(0)\left[\frac{\mathcal{W}(t)-1}{2}\right], \\
\hat{b}_{2}^{\dagger}(t) & =i \frac{\sqrt{2}}{2} \hat{\alpha}(0) \mathcal{Z}(t)+\hat{b}_{1}^{\dagger}(0)\left[\frac{\mathcal{W}(t)-1}{2}\right] \\
& +\hat{b}_{2}^{\dagger}(0)\left[\frac{\mathcal{W}(t)+1}{2}\right],
\end{aligned}
$$

where,

$$
\begin{gathered}
\mathcal{W}(t)=\cosh (\sqrt{2 n} g t), \\
\mathcal{Z}(t)=\sinh (\sqrt{2 n} g t) .
\end{gathered}
$$

In the next topic we calculate the symplectic invariants following the entanglement dynamics of the system.

\section{Dynamics of the Entanglement Between Two NEMS}

Applying the separability criteria on bipartite systems $[15,8]$, in the equation $(4), \eta_{-}$, the state is separable if $\eta_{+} \geq \eta_{-} \geq \frac{1}{2}$. The state is entangled if $0<\eta_{-}<\frac{1}{2}$. In order to calculate the local symplectic invariants (eq. 3 ) with the equation (12), assuming the initial thermal state to NEMS, with thermal numbers $N_{1}=N_{2}=N$, given by

$$
\begin{aligned}
I_{1}=I_{2} & =\left[(\bar{n}+N) \frac{\mathcal{Z}^{2}}{2}+N+\frac{1}{2}\right]^{2}, \\
I_{3} & =\left[\frac{4(N+1)^{2}+2 \bar{n}(N+1)}{4}\right] \mathcal{Z}^{4}, \\
I_{4} & =2 I_{1}\left|I_{3}\right|,
\end{aligned}
$$

the symplectic eigenvalue (eq. 4) results

$\eta_{-}=\left|N+\frac{1}{2}+\frac{\mathcal{Z}^{2}}{2}\left(\bar{n}+N-\sqrt{4(N+1)^{2}+2 \bar{n}(N+1)}\right)\right|$,

Note that, from figure (2), the entanglement dynamics between the two NEMS, give for $\bar{n} \approx N$ and on for 


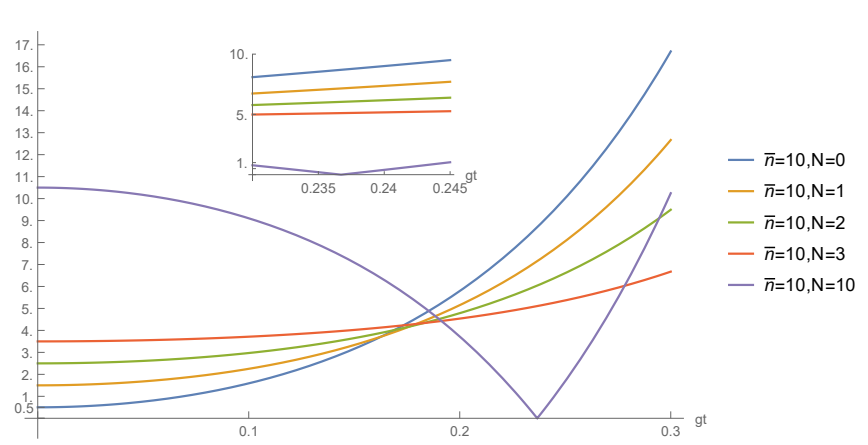

(a)

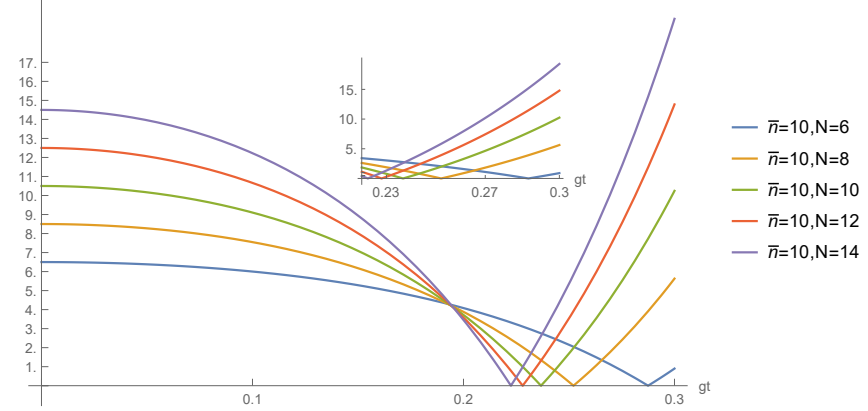

(b)

Fig. 2 The $\eta_{-}(g t)$ for initial states being thermal states. For values of $\bar{n}$ very different from $N$ we have no entanglement as shown in (a). For values close between $\bar{n}$ and $N$, times temporarily bipartite entanglement between the NEMS, and can even instantaneously generate EPR state.

a bounded time interval $T_{e}$ in:

$$
\begin{aligned}
& \frac{\arcsin \left[\sqrt{\frac{2 N}{\sqrt{4(N+1)^{2}+2 \bar{n}(N+1)}-\bar{n}-N}}\right]}{g \sqrt{2 \bar{n}}} \\
& \frac{\arcsin \left[\sqrt{\frac{<T_{e}<}{\sqrt{4(N+1)^{2}+2 \bar{n}(N+1)}-\bar{n}-N}}\right]}{g \sqrt{2 \bar{n}}}
\end{aligned}
$$

and may even generate an $\operatorname{EPR}$ state $\left(\eta_{-}=0\right)$. That is, this model shows us that the TLR can be a means of control for the generation of entanglement between the NEMS.

\section{Proposed of Measure}

The experiment is performed as shown in figure (3). The TLR acts as the source of the controlled entanglement generator. In the sequence, a drive is applied to the TLR, with amplitude $\sqrt{\bar{n}}$. The radiation field there is generated two non-degenerate parametric oscillators. After these interactions, the radiation field can be ejected from the TLR and any correlation can be measured using IQ Mix linear detectors [20]-[21]-[22], giving us the relationship with entanglement quantization

$\left\langle\hat{x}_{\theta}\right\rangle=\left\langle\frac{\hat{\alpha} e^{-i \theta}+\hat{\alpha}^{\dagger} e^{i \theta}}{2}\right\rangle=\sqrt{n\left(1-\mathcal{Z}^{2}\right)} \cos (\theta)$,

because $\mathcal{Z}(t)$ is directly related to the simplectic eigenvalue of equation (14). Other correlations for calculating $\eta_{-}(t)$ can also be made.

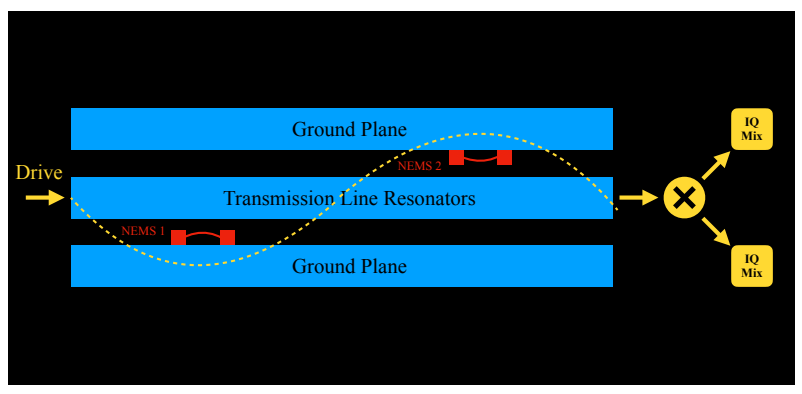

Fig. 3 In the proposed experiment: The output of the TLR is directed to a microwave beam splitter. The two outputs are then amplified and run through separate IQ MIX [21].

\section{Outlook}

In summarized we have demonstrated two central results. The first them, we focus on the behavior of the dynamics of entanglement bipartite in a tripartite circuit using the symplectic eigenvalues $\left(\eta_{-}\right)$extracted from covariance matrix as entanglement parameter. We shown the domain of validity for existence of physical separable state $\left(\eta_{+} \geq \eta_{-} \geq \frac{1}{2}\right)$ and entangled state $\left(0<\eta_{-}<\frac{1}{2}\right)$ via relation of $\bar{n}$ and $N$. The second result is related to entanglement time, when $N \neq 0$ and $\bar{n} \approx N$. We believe that this architecture proposal can be used in development of new circuits to quantum computation and temperature estimation [23]-[24]. This scenario represents the first circuit proposal for entanglement between mechanical thermal states. The other proposals for entanglement between device modes already in the literature are between quantum states and nonlinear interactions [25]-[26]-[27]-[28].

\section{Funding and/or Conflicts of interests/Competing interests}

This work had no funding or conflict of interest. 


\section{References}

1. Brown, Kenton $\mathrm{R}$ and Ospelkaus, Christian and Colombe, Yves and Wilson, Andrew $\mathrm{C}$ and Leibfried, $\mathrm{D}$ and Wineland, David J, Coupled quantized Mechanical Oscillators, Nature 471 (2011) 196-199.

2. Nicacio, F and Furuya, K and Semi FL, Motional Entanglement with Trapped Ions and a Nanomechanical Resonator, Physical Review A 88 (2013) 022330.

3. Gonzalez-Henao, JC and Pugliese, E and Euzzor, S and Abdalah, SF and Meucci, R and Roversi, JA, Generation of Entanglement in Quantum Parametric Oscillators Using Phase Control, Scientific Reports 5 (2015) 13152.

4. Cirac, Juan I and Zoller, Peter, Quantum Computations with Cold Trapped Ions,Physical Review Letters 74 (1995) 4091.

5. Blatt, Rainer and Wineland, David, Entangled States of Trapped Atomic Ions, Nature 453, 1008-1015 (2008).

6. Horodecki, Ryszard and Horodecki, Pawel and Horodecki, Michal and Horodecki, Karol, Quantum Entanglement, Review Modern Physics 81 (2009) 865-942.

7. Haroche, Serge and Raimond, Jean-Michel, Exploring the Quantum: Atoms, Cavities, and Photons, (Oxford university press, 2006).

8. Nicacio, Fernando and de Oliveira, Marcos C, Tight Bounds for the Entanglement of Formation of Gaussian States, Physical Review A 89 (2014) 012336.

9. Wang, Xiang-Bin and Hiroshima, Tohya and Tomita, Akihisa and Hayashi, Masahito, Quantum information with Gaussian states, Physics Reports 448 (2007) 1-111.

10. de Oliveira, Marcos $\mathrm{C}$ and Nicacio, Fernando and Mizrahi, Salomon S, Minkowski Structure for Purity and Entanglement of Gaussian Bipartite States, Physical Review A 88 (2013) 052324.

11. Serafini, Alessio and Illuminati, Fabrizio and Paris, Matteo GA and De Siena, Silvio, Tight Bounds for the Entanglement of Formation of Gaussian States, Physical Review A 69 (2004) 012336.

12. Englert, Berthold-Georg and Wewicz, Krzysztof, Tutorial Notes on One-Party and Two-Party Gaussian States, International Journal of Quantum Information 1 (2003) 153-188.

13. Eisert, $\mathrm{J}$ and Plenio, MB, Introduction to the Basics of Entanglement Theory in Continuous-Variable Systems, International Journal of Quantum Information 4 (2003) 479506.

14. Giedke, G and Cirac, J Ignacio, Characterization of Gaussian Operations and Distillation of Gaussian States, Physical Review A 66 (2002) 032316.

15. Simon, Rajiah, Peres-Horodecki Separability Criterion for Continuous Variable Systems, Physical Review Letters 84 (2000) 2726.

16. Duan, Lu-Ming and Giedke, G. and Cirac, J. I. and Zoller, P., Inseparability Criterion for Continuous Variable Systems, Physical Review Letters 84 (2000) 2722.

17. Paolo Abiuso, Stefan Bl, Daniel Cavalcanti, and Antonio Ac Measurement-Device-Independent Entanglement Detection for Continuous-Variable Systems, Physical Review Letters 126 (2021) 190502.

18. Markus Aspelmeyer, Tobias J. Kippenberg, and Florian Marquardt, Cavity Optomechanics, Review Modern Physics 86 (2014) 1391.

19. Cheng Jiang, Jin-Jin Li Wei He and Ka-Di Zhu, Parametric Coupling Betweena Nanomechanical Ressonator and Superconducting Microwave Cavity, Europhysics Letters 91 (2010) 58002.
20. D. Bozyigit, C. Lang, L. Steffen, J. M. Fink, C. Eichler, M. Baur, R. Bianchetti, P. J. Leek, S. Filipp, M. P. da Silva, A. Blais A. Wallraff, Antibunching of microwave-frequency photons observed in correlation measurements using linear detectors, Nature Physics 7 (2011) 154.

21. Marcus P. da Silva, Deniz Bozyigit, Andreas Wallraff, and Alexandre Blais, Schemes for the observation of photon correlation functions in circuit QED with linear detectors, Physical Review A 82 (2010) 043804.

22. O. P. de Sá Neto, M. C. de Oliveira and G. J. Milburn, Temperature measurement and phonon number statistics of a nanoelectromechanical resonator, New Journal of Physics 17 (2015) 093010.

23. O. P. de á Neto, H. A. S. Costa, G. A. Prataviera and M. C. de Oliveira, Temperature estimation of an entangled pair of trapped ions (arXiv:2109.05008).

24. O. P. de Sá Neto and H. A. S. Costa, Estimation of decoherence in electromechanical circuits, Physics Letters A 383 (2019) 125875.

25. Sh. Barzanjeh, D. Vitali, P. Tombesi, and G. J. Milburn, Entangling optical and microwave cavity modes by means of a nanomechanical resonator, Physical Review A 84 (2011) 042342 .

26. Charles P. Meaney, Ross H. McKenzie, and G. J. Milburn, Quantum entanglement between a nonlinear nanomechanical resonator and a microwave field, Physical Review E 83 (2011) 056202 .

27. O. P. de Sá Neto, M. C. De Oliveira, F. Nicacio, G. J. Milburn, Capacitive coupling of two transmission line resonators mediated by the phonon number of a nanoelectromechanical oscillator, Physical Review A 90 (2014) 023843.

28. V. D. Vaidya, B. Morrison, L. G. Helt, R. Shahrokshahi, D. H. Mahler, M. J. Collins, K. Tan, J. Lavoie, A. Repingon, M. Menotti, N. Quesada, R. C. Pooser, A. E. Lita, T. Gerrits, S. W. Nam and Z. Vernon, Broadband quadraturesqueezed vacuum and nonclassical photon number correlations from a nanophotonic device, Science Advances 6 (2020) 1. 